Proc. Indian Acad. Sci. (Earth Planet. Sci.), Vol. 89, Number 2, July 1980, pp. 267-275.

(C) Printed in India.

\title{
The possible influence of curvature and rotation on ocean currents
}

\author{
R NARASIMHA \\ Department of Aeronautical Engineering, Indian Institute of Science, \\ Bangalore 560 012, India
}

MS received 14 February 1980 ; revised 10 June 1980

\begin{abstract}
Recent laboratory investigations have shown that rotation and (streamwise) curvature can have spectacular effects on momentum transport in turbulent shear flows. A simple model that takes account of these effects (based on an analogy with buoyant flows) utilises counterparts of the Richardson number $\mathrm{Rg}$ and the Monin-Oboukhov length. Estimates of $\mathbf{R g}$ for meanders in ocean currents like the Gulf Stream show it to be of order 1 or more, while laboratory investigations reveal strong effects even at $|R g| \sim 0 \cdot 1$. These considerations lead to the conclusion that at a cyclonic bend in the Gulf Stream, a highly unstable flow in the outer half of the jet rides over a highly stable flow in the inner half. It is conjectured that the discrepancies noticed between observation and the various theories of Gulf Stream meanders, and such phenomena as the observed detachment of eddies from the Gulf Stream, may be due to the effects of curvature and rotation on turbulent transport.
\end{abstract}

Keywords. Turbulent flows; effect of curvature and rotation; ocean currents; Gulf Stream meanders.

\section{Introduction}

A number of laboratory investigations in recent years have shown how turbulence, very possibly because of the quasi-ordered motion contained in it, is greatly affected by a variety of apparently small perturbations. Indeed, the effects can be specta. cular, and may even lead to a total supression of turbulent transport (Narasimha and Sreenivasan 1979). Among such perturbing influences are flow curvature and solid-body rotation. It is the relevance of these findings to geo-physical currents, particularly in the ocean, that we shall discuss in this paper. These effects do not appear to have been considered in standard accounts of turbulence in geophysical systems (e.g. Long 1977).

The next two sections highlight some relevant results of the laboratory research; $\S 4$ will discuss their application to ocean currents. 


\section{Effect of curvature}

Streamline curvature has a surprisingly large effect on the structure and dynamics of a turbulent shear flow. For example, Bradshaw (1969) notes that in a turbulent boundary layer of thickness $\delta$ and curvature $\kappa$, there is a $10 \%$ change in mixing length even when $\kappa \delta$ is only $1 / 300$ !

The nature of the effect of curvature depends on the magnitude and relative sense of curvature with respect to vorticity. It is convenient to speak of a flow as having 'co-vortical curvature' or more simply as 'co-curving' (Narasimha and Sreenivasan 1979) when the two quantities have the same sense; and as "anticurving' in the opposite case (see figure 1).

Simple arguments show that co-curving flows are relatively stable. Thus, in the boundary layer on a convex surface (top left hand configuration in figure 1), consider a slow lump of turbulence thrown outwards. At its new position farther out, the lump has a lower centripetal acceleration than the faster ambient fluid, and so is driven back by the normal pressure gradient, which must be large enough to balance the (faster) mean flow. Similarly, a faster lump of fluid moving towards the surface is thrown out. Turbulent motions therefore tend to be suppressed in such co-curving flows. Anti-curving flows are correspondingly destabilized, and indeed give rise to the well-known Taylor-Görtler vortices aligned with the flow.

Some recent experimental studies in the laboratory show the nature and magnitude of these effects; the suppression of turbulence in co-curving flows has in particular been discussed at length by Narasimha and Sreenivasan (1979). Figure 2, based on the experimental data of Shivaprasad (1976), shows the large differences in momentum transport generated by curvature; the experimental points here are hot wire measurements of the Reynolds shear stress in the boundary layer on the two walls (one convex and one concave) of a curved channel, with $|\kappa \delta| \doteq 0.015$. The distance over which the boundary layer has developed is practically the same in both cases, but the Reynolds shear stress near the concave wall is almost twice that near the convex wall. Furthermore, there is considerably

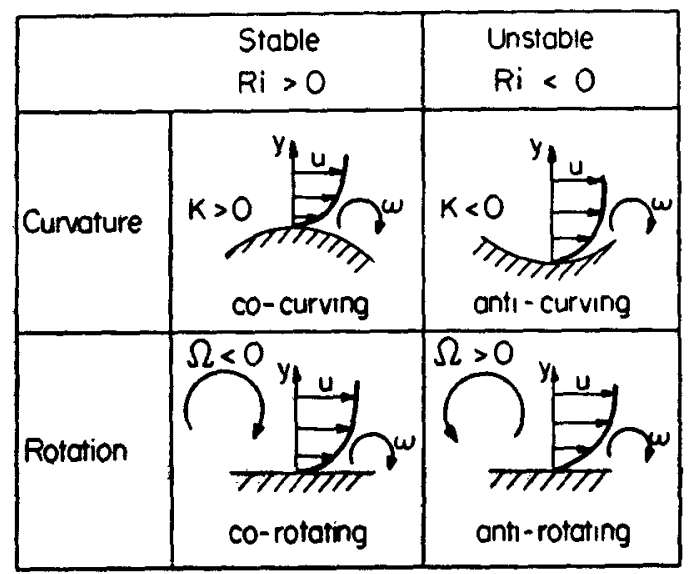

Figure 1. Definitions and sign conventions in curved and rotating flows. 
more mixing in the anti-curving flow, making the concave boundary layer much thicker. So and Mellor (1973) found that the Reynolds shear stress vanished at a distance $y \doteqdot 0.45 \delta$ from the surface in a convex boundary layer of $\kappa \delta \doteq 0.074$; beyond this point the Reynolds stress changed sign (- leading to 'negative' viscosity, if one wishes to put it that way).

One other example of the large effect of curvature should suffice. Figure 3 shows the spectra of the normal velocity fluctuation at $y=0.6 \delta$ in curved boundary layers. Note how energy in the low wave numbers is suppressed in the convex flow and enhanced in the concave flow.

An analogy between buoyant and curved flows has been drawn for a long time; in recent years, Bradshaw (1973) has made extensive use of this analogy to calculate curved turbulent flows. In particular, for a curved shear flow with the mean velocity profile $u=u(y)$ (the longitudinal component of velocity as a function of distance normal to the surface), he defines a 'gradient Richardson number' due to curvature

$$
\operatorname{Rg}_{o}=\frac{2 \kappa^{2} u \partial\left(u \kappa^{-1}\right) / \partial y}{(\partial u / \partial y)^{2}},=2 S_{o}\left(1+S_{o}\right),
$$

where $S_{0} \equiv \kappa u /(\partial u / \partial y)$,

is a non-dimensional flow curvature parameter; clearly $\mathrm{Rg}_{0} \doteq 2 S_{0}$ for small $S_{0}$. Values of $\mathrm{Rg}_{o}$ in the experiments mentioned above are also quoted in figures 2

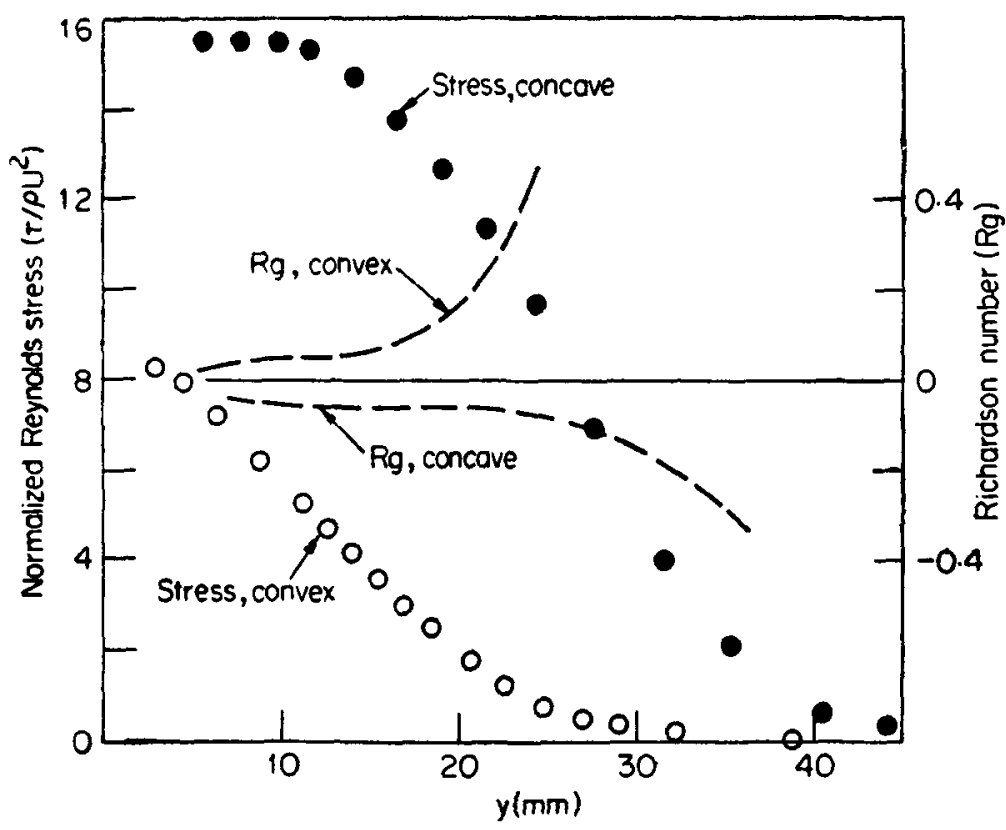

Figure 2. Measured Reynolds shear stress in curved turbulent boundary layers, with curvature parameter $|\kappa \delta| \doteq 0.015$ (data from Shivaprasad 1976). $U$ here is the value of the surface velocity as calculated in potential flow in the curved channel. 


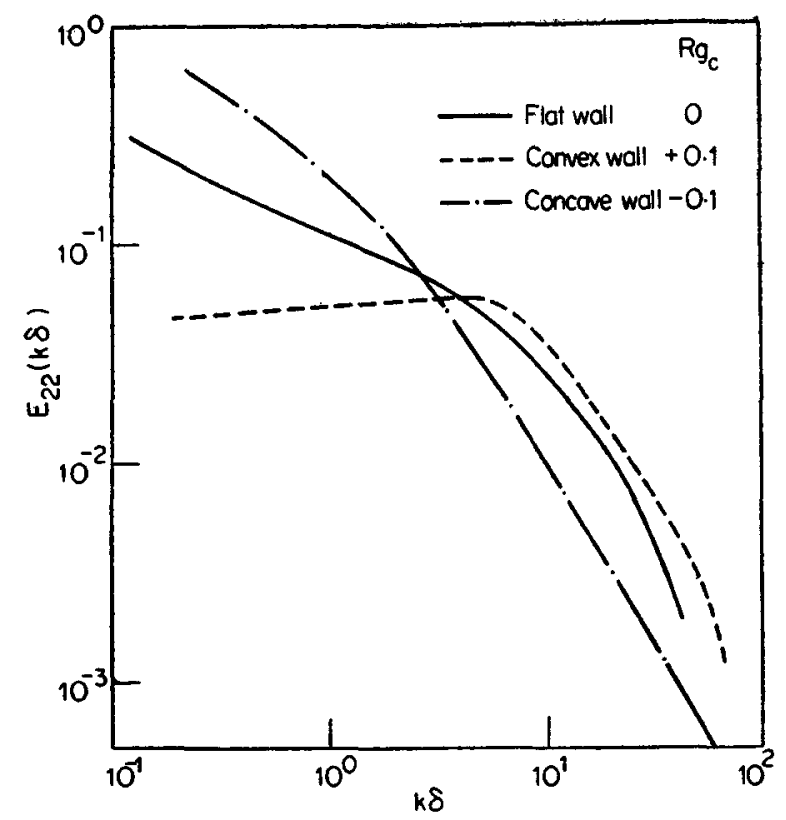

Figure 3. Spectra of the normal velocity fluctuation $v^{\prime}$ in curved boundary layers (data from Shivaprasad 1976). $k$ is the wave number; the spectra are normalized so

that

$$
\int_{0}^{\infty} E_{22}(k \delta) d(k \delta)=1
$$

and 3. Bradshaw suggests that the mixing length $l_{0}$ is altered, in the presence of curvature, to the value $l_{\kappa}$ given by

$$
l_{\kappa}=l_{0}\left(1-\beta_{0} S_{0}\right)
$$

where $\beta_{c}$ is a parameter for which Johnston (1976) suggests the value $\beta_{c} \doteq 6 \pm 2$. One may infer that at a critical value for the Richardson number of about $+\frac{1}{6}$ turbulence is totally suppressed. However, in the shear flows of the type being considered $\mathrm{Rg}$ varies appreciably across the layer (e.g. figure 2), and turbulent transport may maintain energy even when the net local production of turbulent energy is negligible or even negative.

\section{Effect of rotation}

Consider again a shear flow with velocity profile $u=u(y)$ subjected to a solid body rotation $\Omega$. Apart from the well-known Ekman effect, which arises when the vorticity $(\underset{\sim}{\omega})$ is normal to the (imposed solid body) rotation $\underset{\sim}{\Omega}$, there is a strong effect on turbulent transport when the rotation and vorticity are parallel. Detailed experimental studies have only been made of the (fully developed) flow in an effectively two-dimensional channel, rotated about a spanwise axis (Johnston et al 1972). The coriolis force in this situation has a stabilizing or destabilizing influence, depending on whether the rotation has the same or opposite sense to the vorticity. Using the same kind of nomenclature as in curved flows, we may 
therefore again speak of 'co-rotating' and 'anti-rotating' flows (see definition sketch in figure 1). Again as in the discussion of curvature in the previous section, one may see by a simple physical argument that a slow lump of turbulence thrown outwards in a co-rotating boundary layer is driven back by the pressure balancing the relatively higher mean coriolis force. A co-rotating flow is stable, and an anti-rotating flow unstable.

Figure 4 shows how rotation alters the wall stress $\tau_{w}$ in a channel (subscript zero denotes the value in the absence of rotation). It is clear that the stresses are higher on the unstable anti-rotating side; we may expect the corresponding boundary layer to be thicker, but the data of Halleen and Johnston (1967, e.g. figures $4 \cdot 20 \mathrm{a}, \mathrm{b})$ show that this effect is at best rather weak in a channel. It is likely however that in a free flow (like a jet), without the constraining presence of a wall, this effect would be much more pronounced.

The effect of rotation can again be taken into account through a rotation Richardson number

$$
\operatorname{Rg}_{r}=\frac{2 \Omega(2 \Omega-\partial u / \partial y)}{(\partial u / \partial y)^{2}},=S_{r}\left(1+S_{r}\right)
$$

where $S_{r} \equiv 2 \Omega /(\partial u / \partial y)$

is a kind of local inverse Rossby number. The mixing length depends on $\mathrm{Rg}_{\text {r }}$ the same way as it does on $\mathrm{Rg}_{c}(2)$. Indeed, Johnston (1976) suggests that in a curved, rotating flow with $S_{c}, S_{r}$ small,

$$
l=l_{c}\left(1-\beta_{c} S_{c}-\beta_{r} S_{s}\right)
$$

with $\quad \beta_{o} \simeq \beta_{r} \simeq 6 \pm 2$.

\section{Ocean currents}

Consider now an ocean current like the Gulf Stream, as it travels first along the east coast of the United States and then meanders off into the Atlantic. Where the current is relatively straight, we see from the discussion in $\S 3$ that the effect of the earth's rotation is to stabilize the shoreward (co-rotating) side of the

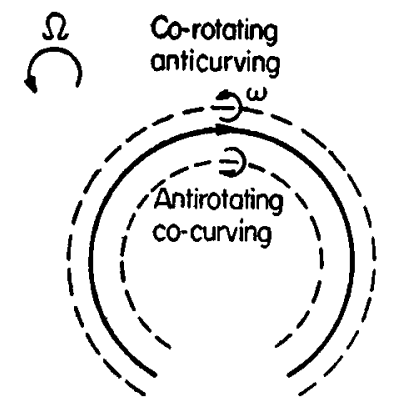

Anticyclonic bend

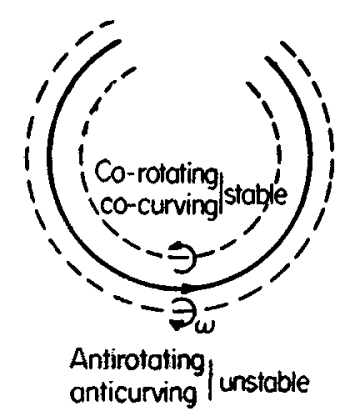

Cyclonic bend

Figure 4. Wall stress in rotating channel flow (data from Johnston et al 1972), $U_{\mathrm{av}}$ is the section-average velocity across the channel. 
current, and destabilize the seaward side (figure 5). It is easily seen that a southern-moving ocean current on an eastern coast in the Southern hemisphere will also be stabilized on the shoreward side and destabilized on the seaward side. It is well known that as the Gulf Stream leaves the US coast near Cape Hatteras, it develops pronounced meanders (Fuglister 1963; Stommel 1965); eventually, as a wide variety of many interesting satellite observations in recent years have shown (e.g. Stumpf and Krishna Rao 1975), some of these meanders pinch off and appear as detached rings* or eddies in the ocean.

These meanders have been generally attributed to the dynamic effect of bottom topography on a current in a rotating inviscid fluid (Warren 1963; Robinson and Gadgil 1970). In an interesting comparison of various theories with observation, Hansen (1970) finds qualitative agreement, but concludes that "the forcing effect of bathymetric variation experienced along the stream path is too weak to account for the curvature amplitudes observed in the meanders."

The present proposal is that the effect of curvature and rotation on momentum transport in and across the current may help to explain the observed discrepancies. Let us first briefly examine the likely qualitative effects.

To begin with, we may call a bend in the current cyclonic or anticylonic, depending on whether the sense of the curvature of the current is the same as or opposite to the sense of the earth's rotation. Now in a jet-like flow, the relative vorticity is of opposite signs on either side of the maximum velocity line. We then see from figure 5 that in an anti-cyclonic bend, the outer region of the current (i.e. the part outside the maximum velocity line) is co-rotating and anticurving, and the inner region is anti-rotating and co-curving. In a cyclonic bend, on the other hand, the outer region is anti-rotating and anti-curving, and the inner region is co-rotating and co-curving. It follows therefore that in an

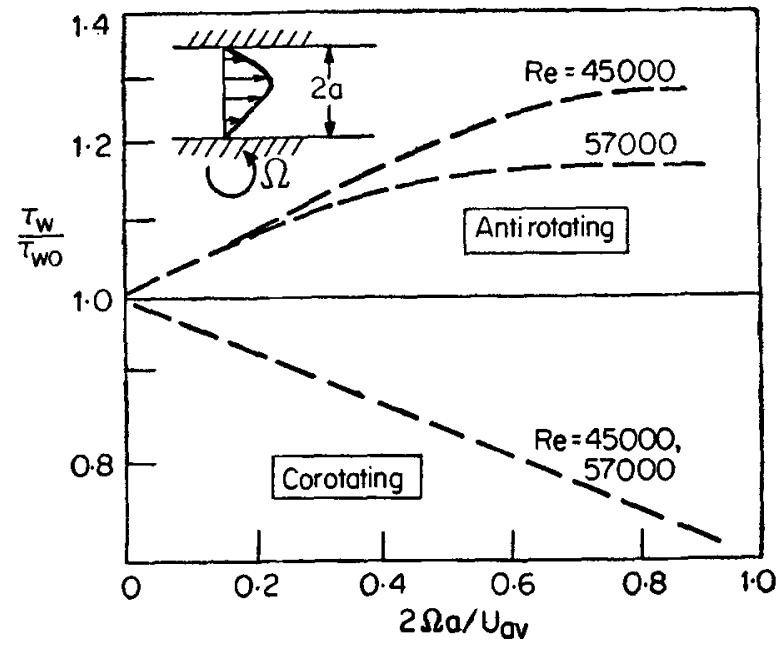

Figure 5. The sense of curvature and rotation effects in an ocean current in the northern hemisphere. ( $\omega$ is the relative vorticity in the current.)

* The term 'ring' has been introduced to distinguish the eddies formed by the breakoff of the Gulf Stream from eddies of other size or origin (Cummings et al 1979). 
anti-cyclonic bend the effects of curvature and rotation oppose each other, whereas in a cyclonic bend they reinforce each other. Furthermore, the outer part of the flow in a cyclonic bend is highly unstable, and the inner part is highly stable: thus there can be a strong stability gradient or eddy viscosity stratification across a current with cyclonic curvature.

It is now necessary to assess the magnitude of these effects. Unfortunately simultaneous measurements of the curvature of the Gulf Stream and the velocity distribution across it do not appear to be available, but the order of magnitude of the parameters involved can be easily estimated.

Some velocity profiles across the current are given by Stommel (1965). Figure 32 of Stommel's book shows a maximum velocity of about $2.5 \mathrm{~m} / \mathrm{s}$, dropping to zero in a distance across the current of 20 to $40 \mathrm{n} . \mathrm{m}$. ( $\simeq 37$ to $74 \mathrm{~km}$ ). The same diagram shows a 'streaky, banded nature of the Stream ... (which) is probably not real ' (Stommel 1965, p. 56). Thus $\partial u / \partial y$ across the current reaches values of the order of $0.5 \times 10^{-4} \mathrm{~s}^{-1}$. A second profile (figure 38) shows a maximum velocity of about $1.5 \mathrm{~m} / \mathrm{s}$; the shear here is much larger on the shoreward than on the sea-ward side, reaching values of about $1.5 \times 10^{-4} \mathrm{~s}^{-1}$. We therefore conclude that velocity gradients across the Gulf Stream are of order $10^{-4} \mathrm{~s}^{-1}$.

The radius of curvature varies widely. In the 'sock' shown in figure 30 of Stommel (1965), this radius is about a degree. Contours of the current shown up on satellite pictures (see e.g. the boundaries reproduced in figure 6), show sometimes a radius of curvature which under extreme conditions may be as low as half-a-degree, or about $50 \mathrm{~km}$. With maximum velocities going up to $2.5 \mathrm{~m} / \mathrm{s}$, $\kappa u$ therefore reach values of about $0.5 \times 10^{-4} \mathrm{~s}^{-1}$.

The normal component of the earth's angular velocity at the latitudes of interest is nearly $10^{-4} \mathrm{~s}^{-1}$.

We thus see that $S_{r}$ is of order 2 in the Gulf Stream, and that $S_{\theta}$ is comparable but probably generally less, reaching values of about 0.5 in a sharply curved region. From (1), (3), the corresponding gradient Richardson numbers are $\mathbf{R g}_{0} \simeq 1.5$ and $\mathrm{Rg}_{r} \simeq 6$. These values are an order of magnitude larger than those at which strong effects have been reported in laboratory studies as discussed in $\S \S 2,3$. There can thus be no doubt that curvature and rotation should be have very strong effects on the structure of the Gulf Stream. (Although it may appear from these numbers that the effects of rotation should be stronger than those of curvature, we must remember that the values of $\mathbf{R g}_{c}$ and $\mathbf{R g}_{r}$ will show appreciable variations both across and along the Stream, and their relative importance would also vary correspondingly.)

We may now examine some features of the Gulf Stream in the light of th above facts. Where the current is still following the coast and is relatively straight, we may expect larger mixing and hence lower vorticity on the (unstable) sea-ward side, compared to the (stable) shoreward side. This asymmetry in the vorticity distribution is indeed observed (Stommel 1965).

We conjecture here that the well-known appearance of 'eddies' near the Gulf Stream may be connected with the effects of curvature and rotation. In particular, as the inner and outer parts of the current on a cyclonic bend are respectively highly stable and unstable, we may expect that the evolution of 
'socks' and the pinching off of an eddy occurs in such bends. Figure 6 shows Gulf Stream boundaries on various days in June 1978, as located (using satellites) by prominent sea-surface temperature gradients, or by the $15^{\circ} \mathrm{C}$ isotherm at $200 \mathrm{~m}$ (NWS 1978). On 7 June we see a prominent cyclonic bend around $65^{\circ} \mathrm{W}$ $37^{\circ} \mathrm{N}$, whose evolution can be traced in the successive maps. On 11 June, the curvature in this bend has become more pronounced at two places, one near $65^{\circ} \mathrm{W} 36^{\circ} \mathrm{N}$ and another near $63^{\circ} \mathrm{W} 39^{\circ} \mathrm{N}$. Note that neither the location nor the curvature of the anticyclonic bend around the westward meander near $65^{\circ} \mathrm{W} 39^{\circ} \mathrm{N}$ has shown any marked change over the same period. By 17 June, eddy $\epsilon$ has pinched off the Gulf Stream, presumably because of the eruption of the cyclonic bend at $63^{\circ} \mathrm{W} 39^{\circ} \mathrm{N}$. Similarly, the cyclonic bend at the southern extreme $\left(\sim 36^{\circ} \mathrm{N}\right)$ is developing into a pronounced sock by 30 June. Data using expendable bathythermographs (NWS 1978) show that this sock did indeed pinch off into a (cold) eddy in July.

One can also see from Figure 6 that, between 17 and 30 June, still another eddy $(\zeta)$ had detached from the stream at another cyclonic bend.

The major point being made is that the formation of both cold (southward) and warm (northward) eddies is attributable to the thrusting movements of the Gulf Stream at cyclonic bends, where a highly unstable outer flow rides on a highly stable inner flow.

It would be worthwhile testing this conjecture by laboratory experiments on curved jets or wakes, by examination of many more ocean current maps, and by computations in which curvature and rotation effects on turbulent transport are appropriately modelled. It is also possible that similar effects play a role in the formation of lows and vortices in the atmosphere.

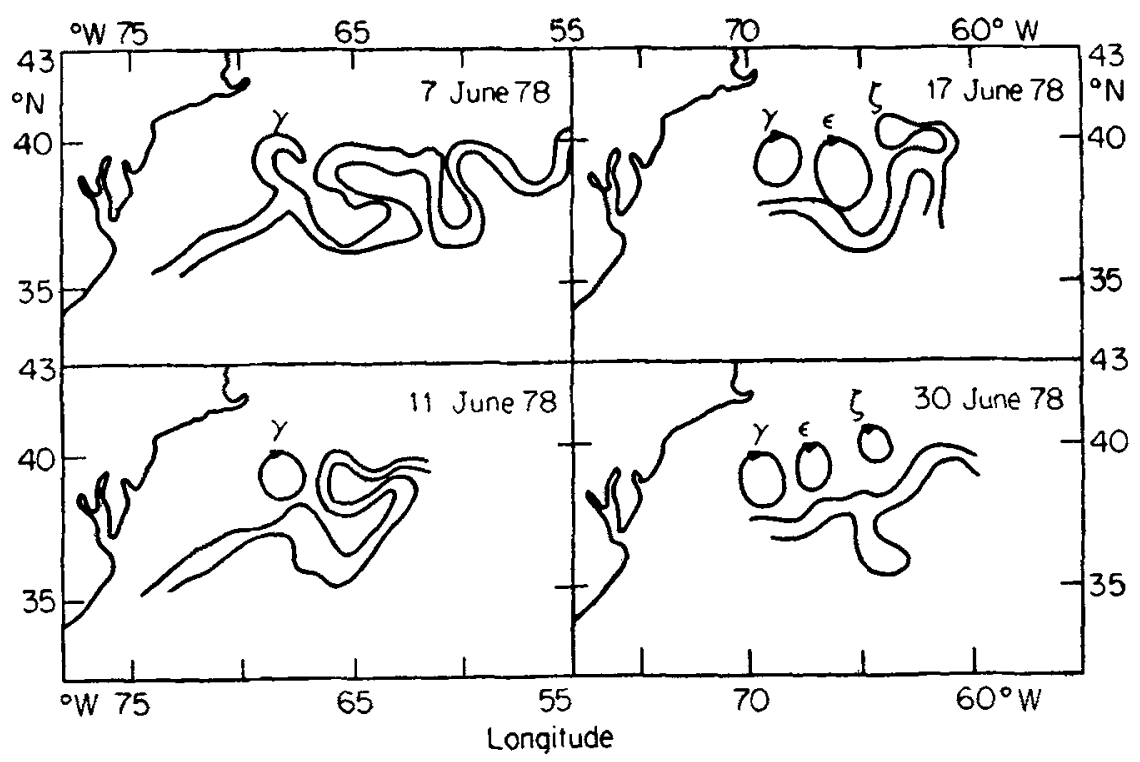

Figure 6. The evolution of meanders and detachment of eddies in the Gulf Stream during June-July 1978. (Based on data published in NWS 1978.) 


\section{Acknowledgements}

The author is indebted to Dr S Gadgil for many interesting discussions on the subject of the paper.

\section{References}

Bradshaw P 1969 J. Fluid Mech. 36177

Cummings B B, Gotthardt G A and Barrett R L 1979 Gulf stream 5 No. 8

Fuglister F C 1963 Prog. Oceanogr. 1263

Halleen R M and Johnston J P 1967 Report MD 18, Thermosciences Division, Dept. Mech. Engg. Stanford Univ., Stanford, Calif.

Hansen D V 1970 Deep Sea Res. 17495

Johnston J P 1976 in Turbulence ed. P Bradshaw (Berlin : Springer Verlag)

Johnston J P, Halleen R M and Lezius D K 1972 J. Fluid Mech. 56533

Laufer J 1975 Annu. Rev. Flu. Mech. 7307

Long R R 1977 Adv. Appl. Mech. 171

Narasimha R and Sreenivasan K R 1979 Adv. Appl. Mech. 19221

National Weather Service, 1978 Gulf stream Vol. 4, May, June, July 1978

Robinson A R and Gadgil S 1970 Geophys Fluid Dyn. 7411

Shivaprasad B G 1976 An experimental study of the effect of ' mild' longitudinal curvature on the turbulent boundary layer, Ph.D. Thesis, Indian Institute of Science, Bangalore

So R M C and Mellor G L 1973 J. Fluid Mech. 6043

Stommel H 1965 The Gulf Stream (Berkeley and Los Angeles : Unive Calif. Press)

Stumpf H G and Krishna Rao P 1975 J. Phys. Oceanogr. 5388

Warren B A 1963 Tellus 15167 